Original Research Paper

\title{
Optimal Arrangement of Energy-Dissipating Members for Seismic Retrofitting of Truss Structures
}

\author{
${ }^{1}$ Toru Takeuchi, ${ }^{2}$ Yusuke Kinouchi, ${ }^{1}$ Ryota Matsui and ${ }^{1}$ Toshiyuki Ogawa \\ ${ }^{I}$ Department of Architecture and Building Engineering, Tokyo Institute of Technology, Japan \\ ${ }^{2}$ Former Graduate Student, Tokyo Institute of Technology, Japan
}

Article history

Received: 21-06-2015

Revised: 22-06-2015

Accepted: 29-06-2015

Corresponding Author:

Toru Takeuchi

Department of Architecture and

Building Engineering, Tokyo

Institute of Technology, Japan

E-mail: ttoru@arch.titech.ac.jp

\begin{abstract}
Although passive response control design is a popular method, an arrangement methodology for energy-dissipating devices in spatial structures has not been established yet. In this study, optimization techniques are applied to arrangements of energy-dissipating devices in truss tower structures. First, a response prediction rule using the response spectrum and the equivalent linearization method is proposed. Then, a genetic algorithm is applied to select the optimal arrangement of viscoelastic damper devices in truss tower structures. Additionally, the device arrangements determined by empirical design methods are compared with the optimal solutions.
\end{abstract}

Keywords: Truss Structures, Response Control, Arrangement Optimization, Genetic Algorithm, Equivalent Linearization Method

\section{Introduction}

Because telecommunication infrastructure plays an important role after huge seismic disasters, it is essential to assure the structural capacity of existing truss tower structures after the earthquake. In recent years, response control seismic retrofitting by substituting members of tower structures with energy dissipating braces has been proposed and applied in a practical case (Fig. 1, Ookouchi et al., 2006). However, an optimal damper design methodology for the response control retrofitting of such free-formed truss structures has not been studied comprehensively yet.

Genetic Algorithms (GAs) are often used to solve structural optimization problems, particularly static analysis problems. A GA is constituted based on the evolution of genes and can easily find the optimal solution through selection, crossing over and mutation processes, even if in the case that the objective function and its sensitivity functions are difficult to mathematically formulate. For example, Hagishita et al. (2008) applied a heuristic optimization method for the layout of the bracings on semi-rigid frames. Young et al. (2014) discussed the optimal arrangement of braces based on the natural period of braced steel frames for seismic retrofitting. However, these studies do not directly evaluate the response reduction effects of each seismic retrofitting. Nakazawa et al. (2006) developed a method for determining the optimal damper arrangement in a truss tower using a GA and a grid computing system based on time-history response analyses. However, this method requires a vast number of analyses and substantial calculation time.

In this study, a method for determining the optimal arrangement of viscoelastic dampers (VEDs) minimizing the top displacement of truss tower structures or the buckling risk of each member with much less calculation time is proposed. The proposed method applies response spectrum evaluation using the equivalent linearization method and an optimization algorithm with a GA. The results of the proposed method are examined and validated with time history analysis. Additionally, the obtained optimal damper arrangements are compared with the damper arrangements determined using the empirical design procedure.

\section{Damper Design Optimization Method for Truss Structures}

\section{Optimization Algorithm}

A Simple Genetic Algorithm (SGA, Holland 1975) treating a single objective index is applied as a sort of optimization algorithms for the structural optimization of truss structures. The tournament method (size: 2 ) is used to choose solutions inherited from previous generations and crossing is applied uniformly to each gene during the optimization process. The crossing and mutation ratios are 0.5 and 0.001 , respectively. 


\section{Formulation of Objective Function of SGA}

The objective function of the SGA is formulated by employing the response spectrum method and the equivalent linearization method. In the analysis, the main structure is assumed to be elastic and each VED is assumed as the Kelvin-Voigt model. The response reduction effects of the VEDs are estimated from the stiffness and damping of entire structure by assuming a proportional damping system. This is based on an assumption that the effect of additional VEDs on the vibration mode of the entire structure is negligible.

An outline of the proposed evaluation method is shown in Fig. 2. The equivalent damping ratio ${ }_{m} h_{e q}$ of the m-th mode is calculated by referring to the eigenvalue analysis results of the three-dimensional (3D) model containing the stiffness of the added VEDs in elastic state. The response reduction effect of the $\mathrm{m}$-th mode is calculated from the damping reduction factor ${ }_{m} D_{h}$ as Kasai et al. (1998).

${ }_{m} D_{h}=\sqrt{\frac{1+a h_{o}}{1+a_{m} h_{e q}}}, \quad(a=25)$

The maximum response is evaluated by synthesizing the response of various modes using the Complete Quadratic Combination (CQC) method (Wilson et al., 1981). In the CQC method, different modes that satisfy over $90 \%$ of the effective mass are adopted for the response prediction (Ogawa et al., 2003).

For multi-storey structures (Takeuchi et al., 2002), the equivalent damping ratio $h_{e q}$ of the entire structure is normally evaluated by considering the elastic strain energy $W_{i}$ and the equivalent damping ratio $h_{\text {eqi }}$ of each storey as in Equation 2. The value is calculated using lateral force distribution based on the first mode response:

$$
h_{e q}=\frac{\sum_{i=1}^{N^{\prime}} h_{e q i} \cdot W_{i}}{\sum_{i=1}^{N^{\prime}} W_{i}} h_{0}+\frac{1}{4 \pi} \cdot \frac{\sum_{i=1}^{N^{\prime}} E_{d i}}{\sum_{i=1}^{N^{\prime}} W_{i}}
$$

$N^{\prime}$ is the number of stories. Applying this relationship to each member, the equivalent damping ratio ${ }_{m} h_{e q}$ of the entire structure can be evaluated by considering the elastic strain energy ${ }_{m} W_{i}$ of each member and the equivalent damping ratio ${ }_{m} h_{\text {eqi }}$ of each VED.

Let us consider by replacing the entire structure with an equivalent Single-Degree-Of-Freedom (SDOF) model as in Fig. 3. If the VEDs and main structure are connected in parallel as in Fig. 3(c), the elastic strain energy $W_{a}$ of the entire structure is the sum of the strain energy of the VEDs and the main structure $\mathrm{W}_{\mathrm{d}}+\mathrm{W}_{\mathrm{b}}$. However, these relationships in the truss structures are assumed to be similar to a serial system, because the surrounding frame deforms by the reaction forces of VEDs.

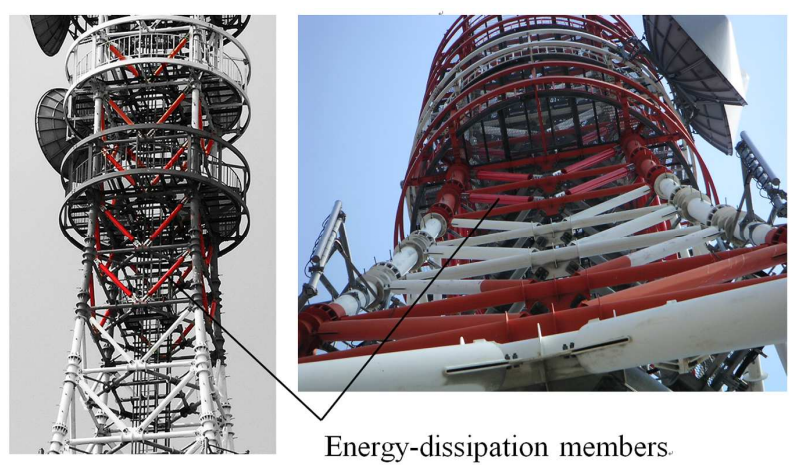

Fig. 1. Seismic retrofitting of truss tower with energydissipating members

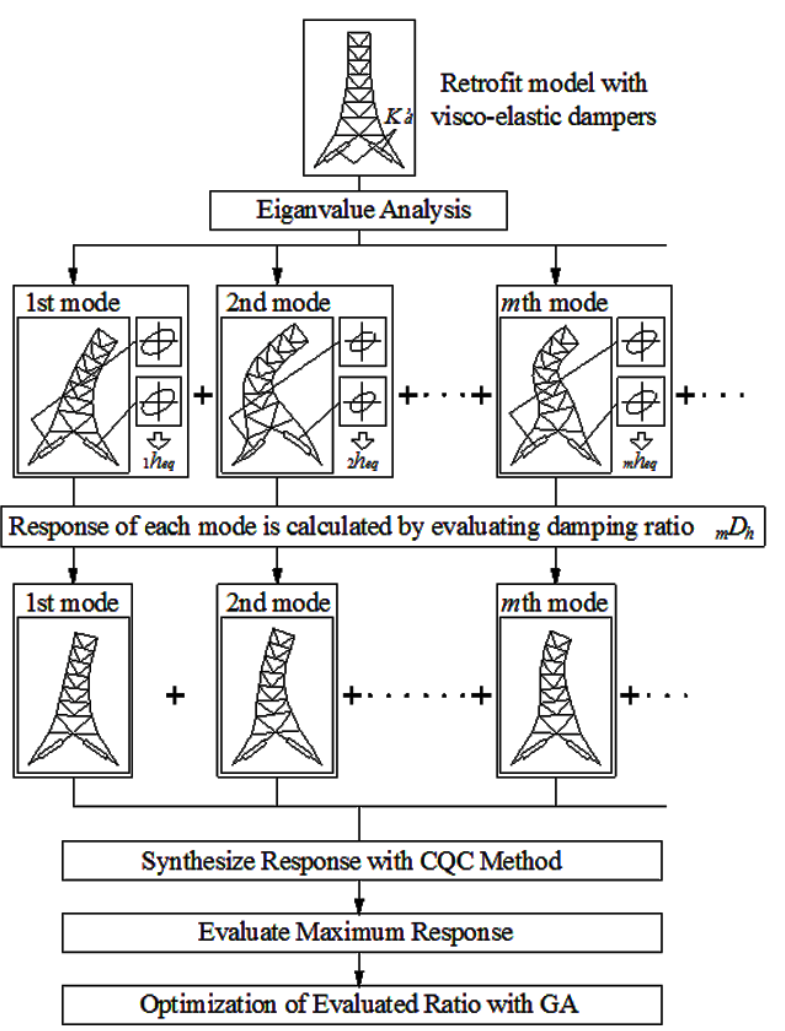

Fig. 2. Proposed evaluation method and supposed hysteresis loop

In the serial system (Fig. 3 (b)), the phase of the displacement between the VEDs and the main structure is slightly shifted because of the viscosity of the VED. The elastic stiffness $K_{a}^{\prime}$ and loss factor $\eta_{a}$ of the entire structure are then calculated as:

$$
\begin{aligned}
& K_{a}^{\prime}=\frac{\left\{\left(1+\eta_{d}^{2}\right) K_{d}^{\prime}+K_{b}\right\} K_{d}^{\prime} K_{b}}{\left(K_{d}^{\prime}+K_{b}\right)^{2}+\left(\eta_{d} K_{d}^{\prime}\right)} \\
& \eta_{a}=\frac{\eta_{d}}{1+\left(1+\eta_{d}^{2}\right) K_{d}^{\prime} / K_{b}}
\end{aligned}
$$




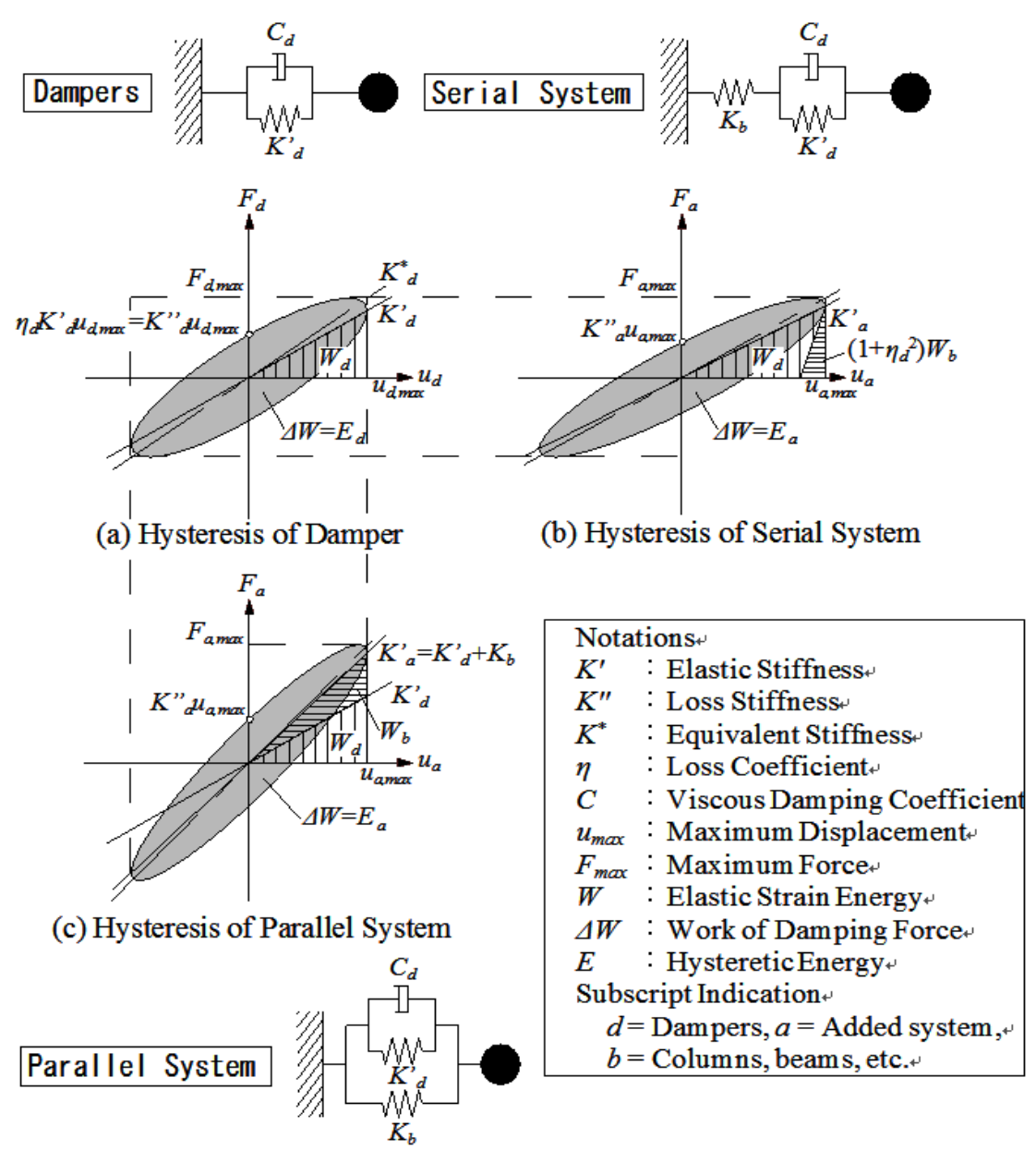

Fig. 3. Relationship between the damper and frame

The elastic strain energy $W_{a}$ of a serial system is calculated from the equality of the force phase and the hysteretic energy between the VEDs and the main structure as:

$W_{a} \frac{1}{2} K_{a}^{\prime} u_{a, \max }^{2}=\left(1+\eta_{d}^{2}\right) W_{b}+W_{d}$

In a Multi-Degree-Of-Freedom (MDOF) model, the equivalent damping ratio ${ }_{m} h_{e q}$ of the $m$-th mode is calculated by applying the abovementioned serial theory to all VEDs. In Equations (6) and (7), ${ }_{m} \omega$ is the fundamental angular frequency and $N$ is the number of members. The maximum response is evaluated by the response spectrum method assuming ${ }_{m} h_{e q}$ :

${ }_{m} h_{e q}=h_{0}+\frac{1}{4 \pi} \cdot \frac{\sum_{i=1}^{N}\left\{{ }_{m} E_{d i} /\left(1+{ }_{m} \eta_{d i}^{2}\right)\right\}}{\sum_{i=1}^{N}{ }_{m} W_{b i}+\sum_{i=1}^{N}\left\{{ }_{m} W_{d i} /\left(1+{ }_{m} \eta_{d i}^{2}\right)\right\}}$

$$
=h_{0}+\frac{1}{4 \pi} \cdot \frac{\sum_{i=1}^{N}\left\{\pi m \omega C_{d i} m u_{d i, \max }^{2} /\left(1+{ }_{m} \eta_{d i}^{2}\right)\right\}}{\sum_{i=1}^{N}{ }_{m} W_{b i}+\sum_{i=1}^{N}\left\{{ }_{m} W_{d i} /\left(1+{ }_{m} \eta_{d i}^{2}\right)\right\}}
$$

\section{Analysis Conditions and Model}

\section{Analysis Conditions}

The time history analysis of a 3D truss tower model is performed to confirm the effectiveness of the proposed method. The structural damping is set to be proportional to the stiffness and the inertia mass of the truss tower is determined by its weight. The Newmark- $\beta$ method is used for the numerical integration $(\beta=1 / 4)$ and the time interval of the analysis is $0.01 \mathrm{sec}$. Geometric nonlinearity is taken into account. Earthquake waves are applied only in the $x$-direction.

\section{Analysis Model}

The analysis model is based on an actual telecommunication truss tower consisting of 15 layers (Fig. 4) placed on a five-storey concrete building 
(Ookouchi et al., 2006). The slenderness ratios of the diagonal members range from 54 to 68 . Although the elastic stiffness of the replaced VED is divided into four categories depending on the installation location, the damping coefficient of all dampers is equal to $C d$ $=78.4 \mathrm{kNsec} / \mathrm{cm} \mathrm{rad}$. Damping ratios of $h_{s u b}=0.03$ and 0.01 are used on the substructure and truss tower respectively, to represent the structural damping. For the simplification of the analysis, the tower model placed on the SRC building is analysed by a converted input motion (1R-3R) as follows.

The ground motion waves are applied to the SRC building with the truss tower and the response acceleration on the truss basement is employed as $1 \mathrm{R}$ $3 \mathrm{R}$ input motion of the truss tower as if it stood on the ground. This assumption is based on the weight of the tower structure is much lighter than supporting building, otherwise the interactive response effects should be taken into account as Papageorgioul and Gantes (2010a,b) indicates. Ground motion waves are Hachinohe EW, JMA Kobe NS and artificial random waves normalized by the Japanese design spectrum.

Separately, the ground motions are directly input to each model which are named as $1 \mathrm{G}-3 \mathrm{G}$.

Here, a small amount of noise in the spectrum should be avoided to obtain optimal solutions. Therefore, the smoothing spectrum obtained by the moving average of the sequence of three values the original acceleration response spectrum $(h=0.05)$ at an interval of $0.05 \mathrm{sec}$ is employed. The dominant modes of the analysis model are shown in Fig. 5 and the acceleration response spectra of the $1 \mathrm{R}$ and $1 \mathrm{G}$ wave (Hachinohe EW) are compared in Fig. 6. This comparison indicates that the dominant natural period of the ground motion and the response wave on top of the SRC building is different. The $1 \mathrm{R}$ wave excites the 4 th vibration mode.

\section{Effectiveness of Proposed Response Prediction Rule}

The effectiveness of the proposed response prediction rule is firstly investigated by comparing the evaluation results to those of the time history analysis in Fig. 7. The $1 \mathrm{R}$ earthquake wave is applied in the $x$-direction.

The maximum response displacement of the retrofitted structure with VEDs decreases to $1 / 4-1 / 3$ times that of the non-retrofitted structure and the difference between the time-history analysis results and those of the proposed prediction rule is less than $15 \%$.

Thus, the proposed prediction rule is considered to be valid. The evaluation assuming a parallel system overestimates the damping ratio in higher modes, whereas the evaluation with a serial system has the safety margin. The calculation time of the response prediction decreases to $1 / 15000$ of the time-history analysis time and the proposed evaluation method is found to be effective to find general optimal solutions.

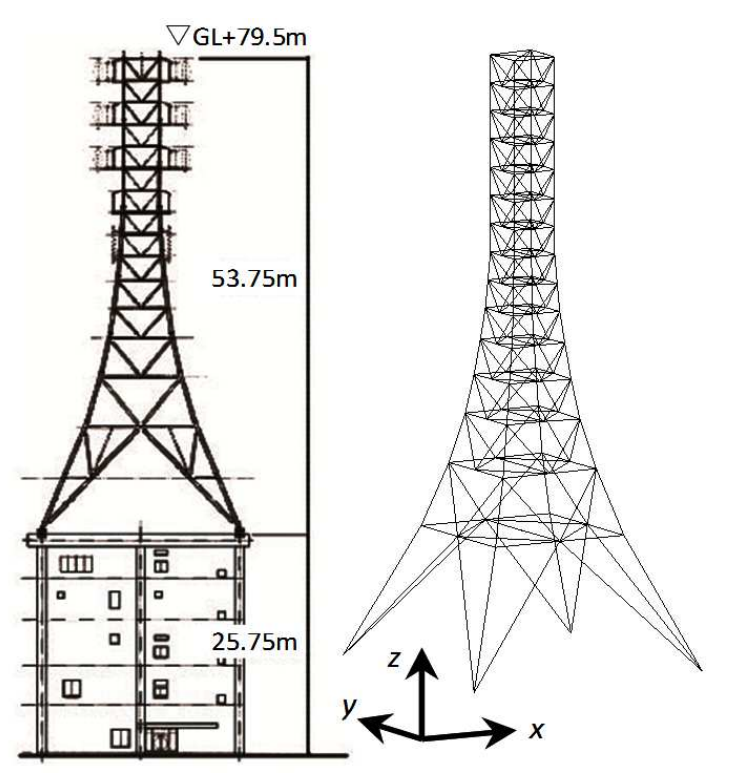

Fig. 4. Analysis model

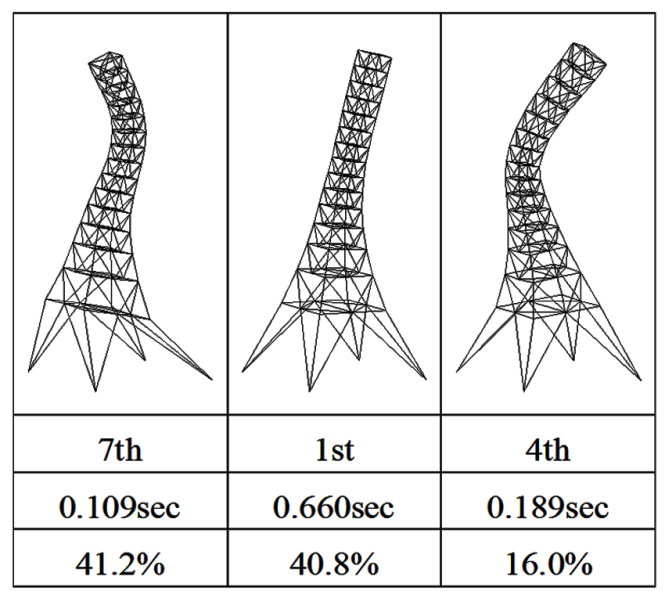

Fig. 5. Dominant vibration modes and effective mass ratios

\section{Optimization Targeting for All Members}

\section{Formulation of Optimization Problem SOP1}

First, SOP1 is represented as follows. The objective function is set as the maximum lateral displacement $\delta_{t o p}$ at the top of the truss structure.

Out of 360 members including main columns, diagonal members, horizontal beams and bracing members, 24 members are selected for replacing to VED. On the condition of the symmetry in the $x z$ and $y z$ planes, the problem is reinterpreted as 6 members selected from 90 members. Therefore, the number of solution combinations for SOP1 is ${ }_{90} \mathrm{C}_{6}=622,614,630$. In the optimization algorithm, the VED arrangement information is coded as 0 (no VEDs exist) or 1 (VEDs exist) for all members on each chromosome. 


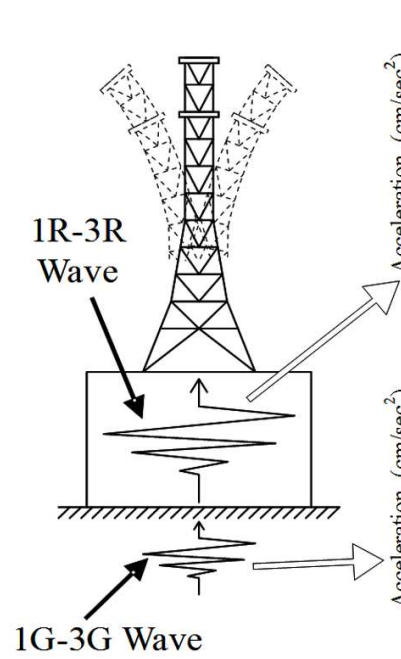

1G-3G Wave

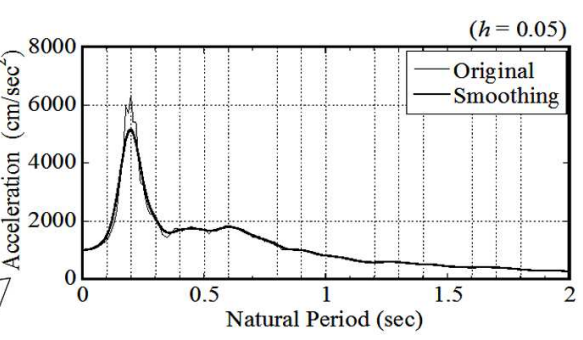

(a) $1 \mathrm{R}$ wave

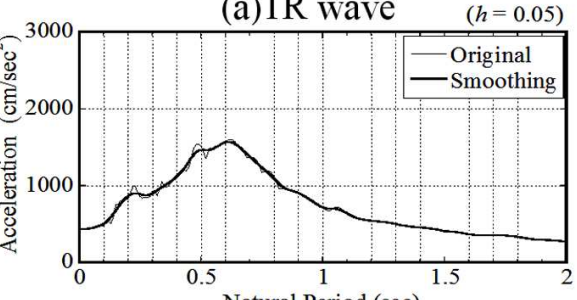

(b) $1 \mathrm{G}$ wave

Fig. 6. (a) $1 \mathrm{R}$ wave (b) $1 \mathrm{G}$ wave Acceleration response spectrum

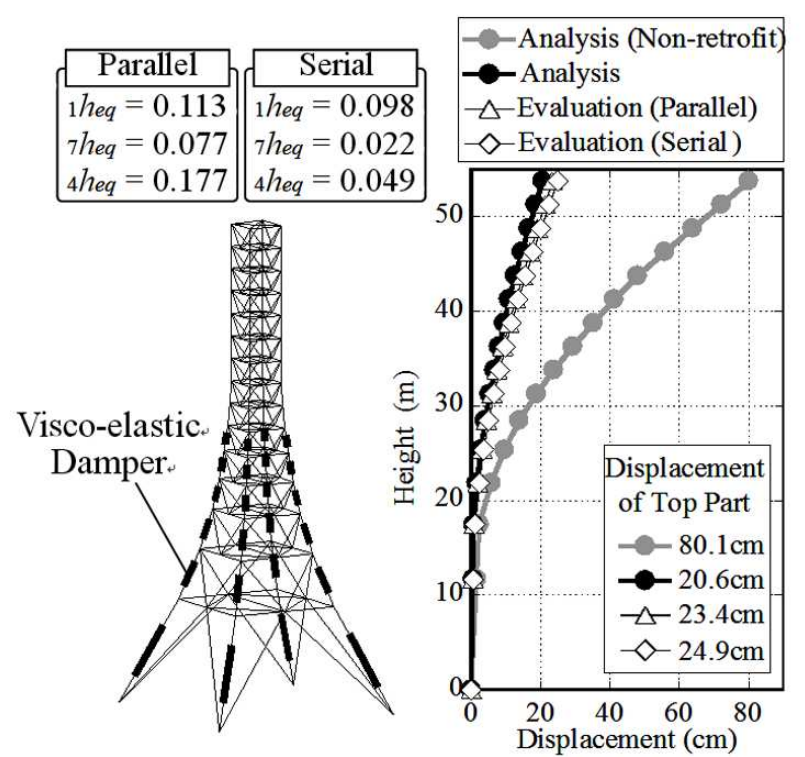

(a) A1 Model

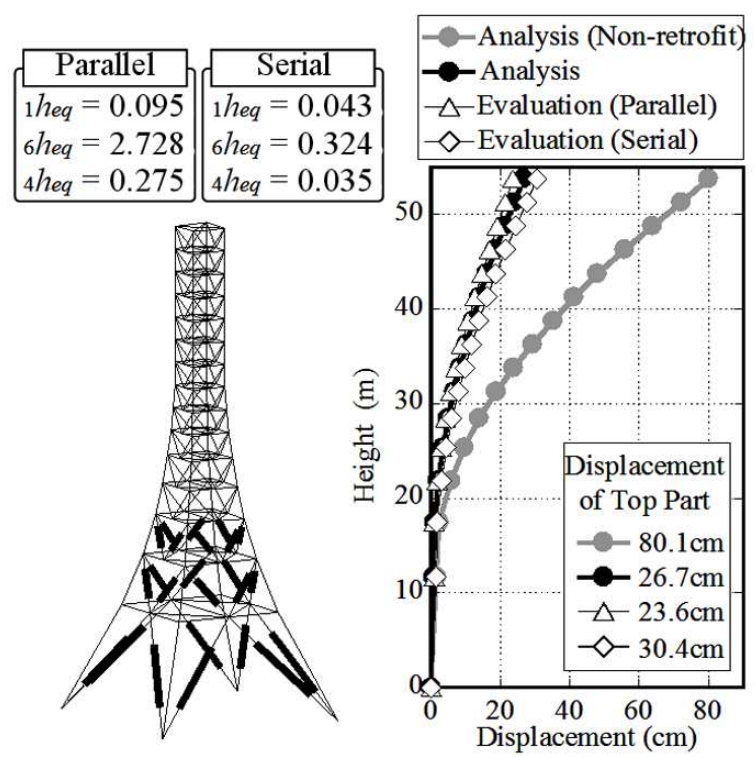

(b) A2 Model

Fig. 7. (a) A1 Model (b) A2 Model Validity of the response evaluation method

\section{Optimal Arrangements in SOP1}

Optimization analysis is conducted three times for each case because the convergence characteristics of the GA are random compared to other algorithms. The population number is 500 and the best solution remaining constant through 150 generations is judged as the convergent optimal solution. Fig. 8 shows the optimal VED arrangement and a comparison of the results (the maximum displacement at the top) between the evaluation and the dynamic analysis results. In each case, the same optimal solution is obtained in 9th-4th generations in the three trials.
The main column members of 1st-3rd layers and the diagonal members in 1st layer are replaced by VEDs in all trials and three types of VED arrangement solutions are obtained. In the cases of $1 \mathrm{R}$ and $3 \mathrm{R}$ motion, the main column member of 9 th layer is selected. In cases $1 \mathrm{G}$ and $3 \mathrm{G}$, the diagonal member in the $y$-direction of 2 nd layer is selected. These solutions differ from the truss tower with the substructure and the self-supported tower. Conversely, the optimal VED arrangements in cases $2 \mathrm{R}$ and $2 \mathrm{G}$ (JMA Kobe NS) coincide. The errors between the evaluation and time history analysis results are approximately $20 \%$ in each case. 


\begin{tabular}{|c|c|c|c|c|c|}
\hline \multicolumn{2}{|c|}{ HACHINOHE-EW } & \multicolumn{2}{|c|}{ JMA-KOBE-NS } & \multicolumn{2}{|c|}{ RANDOM } \\
\hline 1R (On Build.) & $1 \mathrm{G}$ (At Ground) & 2R (On Build.) & 2G (At Ground) & 3R (On Build.) & 3G (At Ground) \\
\hline 17 & 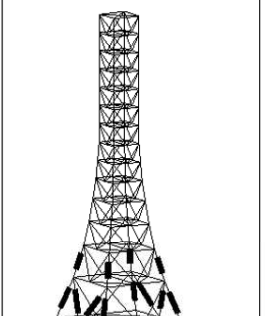 & & . & & 11 \\
\hline $21.5 \mathrm{~cm}$ & $19.0 \mathrm{~cm}$ & $21.2 \mathrm{~cm}$ & $19.3 \mathrm{~cm}$ & $22.4 \mathrm{~cm}$ & $18.6 \mathrm{~cm}$ \\
\hline $18.3 \mathrm{~cm}$ & $14.7 \mathrm{~cm}$ & $16.2 \mathrm{~cm}$ & $15.2 \mathrm{~cm}$ & $20.1 \mathrm{~cm}$ & $16.4 \mathrm{~cm}$ \\
\hline
\end{tabular}

Upper column: Evaluation Result Lower Column: Time History Analysis Result

Fig. 8. Optimal solutions of SOP1

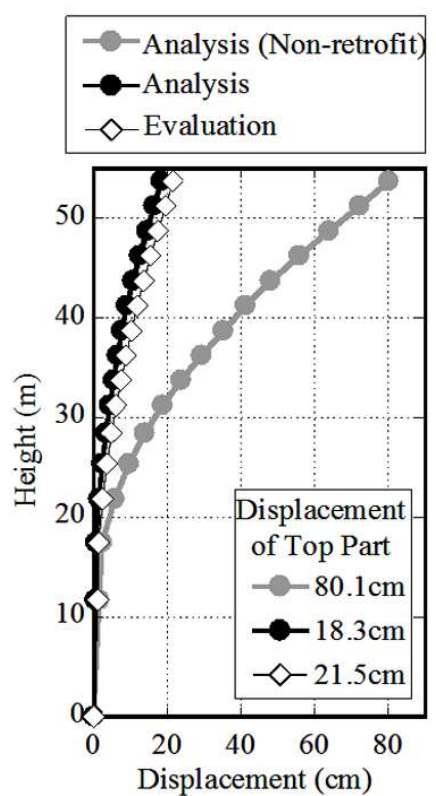

(a) 1R Case

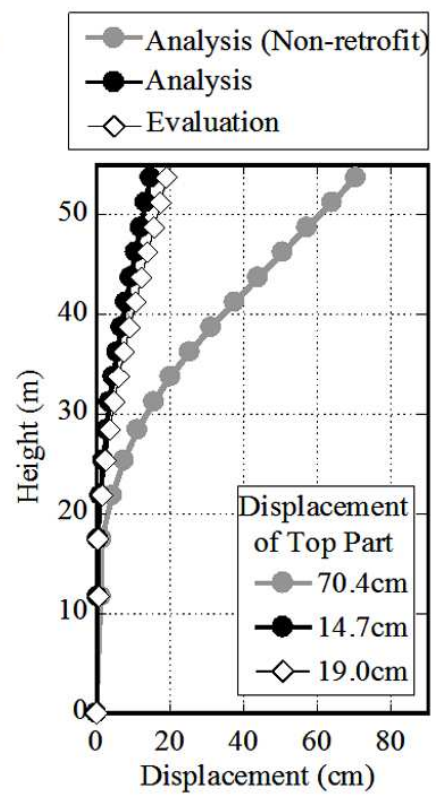

(b) $1 \mathrm{G}$ Case.

Fig. 9. Lateral displacement in SOP1

The response displacement of the optimal solution of $1 \mathrm{R}$ and $1 \mathrm{G}$ motion is shown in Fig. 9. The proposed method largely captures the response of the retrofitted structure of the optimal solution with only minor errors. Additionally, the responses of the optimal retrofitted models decrease to $1 / 4-1 / 3$ times that of the non retrofitted model in each case. Accordingly, the proposed optimization method with a GA is considered to be available to optimize the VED arrangement of the target truss tower structure.

\section{Optimization Targeting for Diagonal Members}

In actual retrofitting construction, it is not practical to replace the main column members to the VED for supporting the vertical load. Therefore, in this chapter, an optimal VED arrangement achieved by replacing only diagonal members is investigated. Because the solution space of the optimization problem is relatively small, a full search for the solution space is also conducted for comparison with the optimization method. 


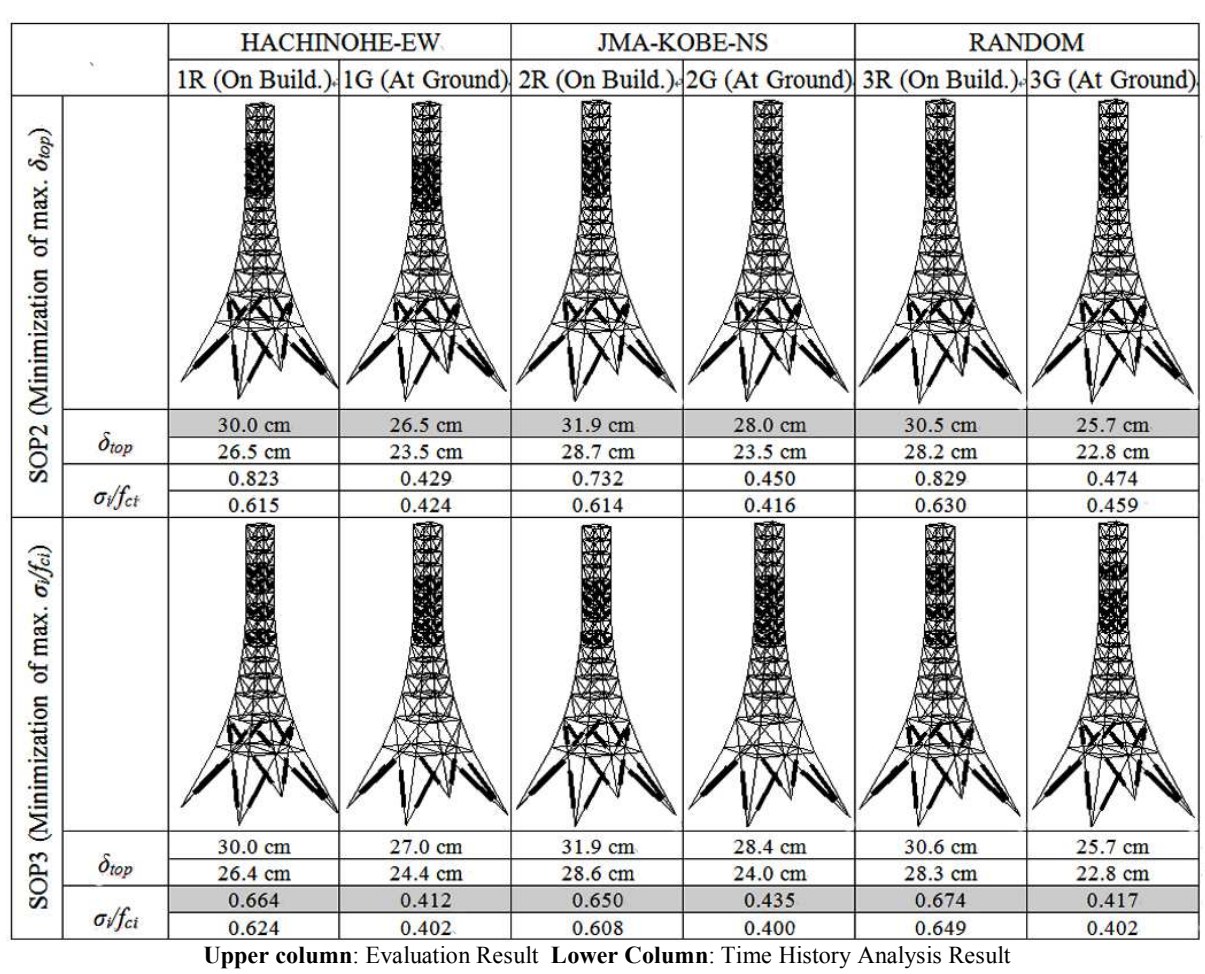

Fig. 10. Optimal solutions of SOP2 and SOP3

\section{Formulation of Optimization Problems SOP2 and SOP3}

The objective values of SOP2 and SOP3 are the maximum displacement of the top $\delta_{\text {top }}$ and minimum buckling risk index, respectively. For SOP3, the risk index is defined as $\sigma_{i} / f_{c i}$, is set as objective function, where $f_{c i}$ is the allowable design buckling stress and $\sigma_{i}$ is the maximum axial stress in each member. The buckling risk index of the VEDs is defined as zero. For SOP2 and SOP3, 48 diagonal members can be replaced to the VEDs from 120 diagonal members. This means that six layers of the tower can be selected under the symmetry condition; therefore, the number of solution combinations is 5,005 .

\section{Optimal Arrangements in SOP2 and SOP3}

Optimization analysis is conducted three times in each case. The population number is 100 and the best solution remaining constant through 150 generations is judged as the convergent optimal solution.

Figure 10 shows the optimal VED arrangements, the maximum displacement at the top $\delta_{t o p}$ and the buckling risk index $\sigma_{i} / f_{c i}$ of each optimal solution. In each case, the same optimal solution is obtained in each of the three trials. The optimal VED arrangements are distributed in the lower part (1st and 2nd layers) and the middle part (7th-12th layers).

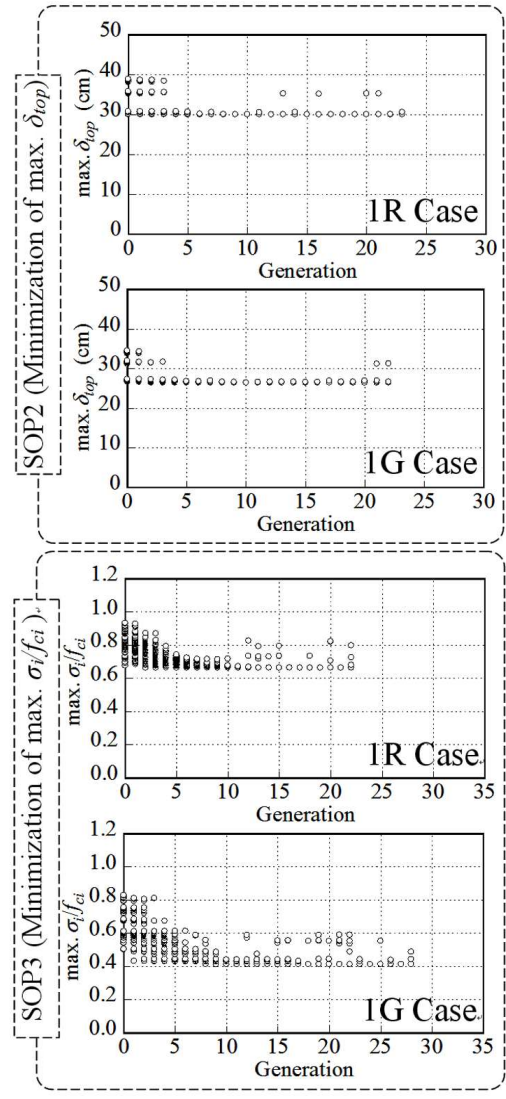

Fig. 11. Transition of fitness ratio 


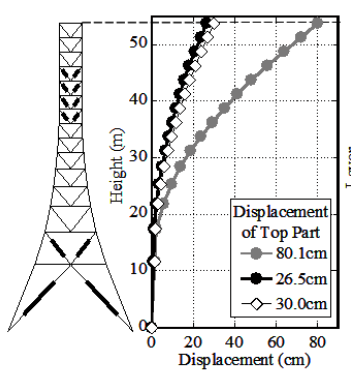

(a) SOP2, 1R Case

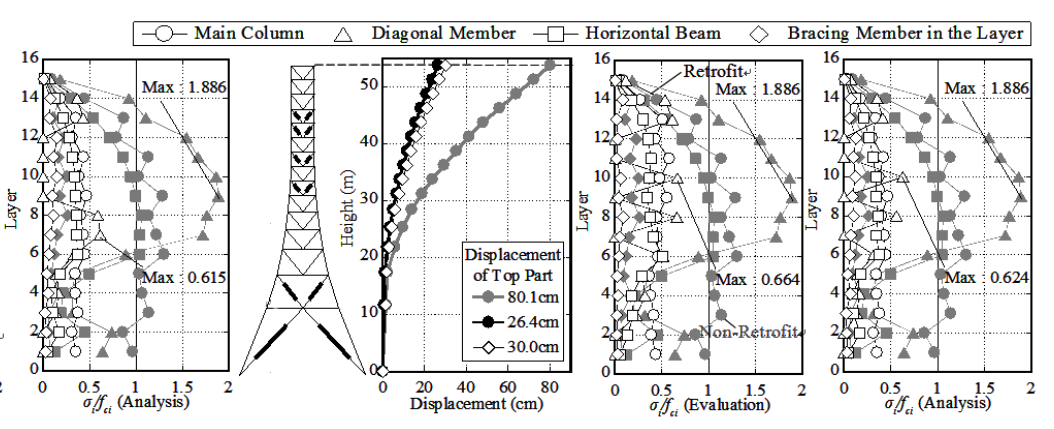

(b) SOP3, 1R Case

Fig. 12. (a) SOP2, Case 1R (b) SOP3, Case 1R Maximum response displacement and buckling risk index

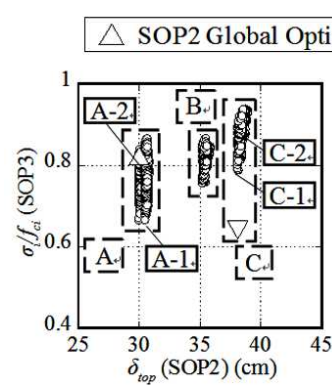

(a1) 1R Case

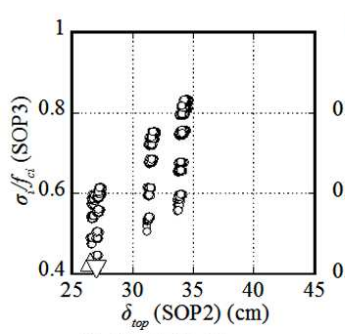

(b1) $1 \mathrm{G}$ Case

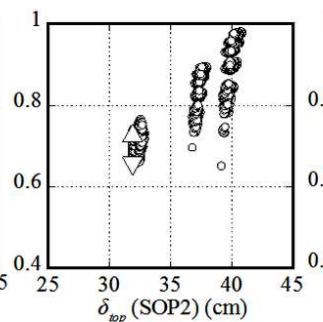

(a2) 2R Case

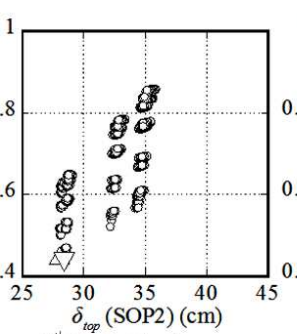

(b2) $2 \mathrm{G}$ Case

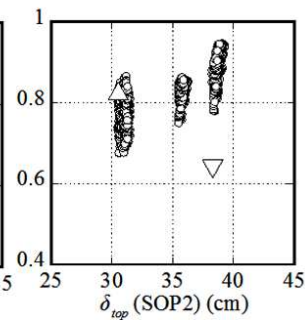

(a3) 3R Case.

Fig. 13. Evaluated values of all solutions

Optimal solutions of SOP2 include only two types of arrangements, in which the VEDs are placed continuously in the middle part of the truss tower. Conversely, the optimal arrangements of SOP3 are different from SOP2. The errors between the evaluation values and time history analysis results in each case are approximately 10\%. Fig. 11 shows the transition of the evaluation values during the optimization calculation. The steps at which optimalsolutions are reached in SOP2 and SOP3 are the 1st-6th and 2nd-11th generations, respectively.

The maximum response displacement and the maximum buckling risk index of case $1 \mathrm{R}$ are shown in Fig. 12. The evaluation value and analysis results agree with each other. The diagonal member or main column of the middle part of the truss tower indicate the maximum buckling risk index among whole structural members and its tendency is almost same in the evaluation values and analysis results in each case.

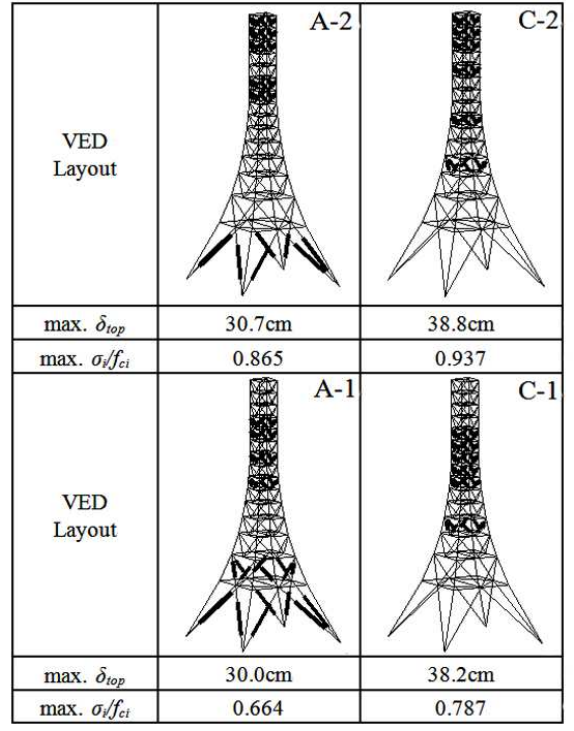

Fig. 14. VED arrangements for specific cases 


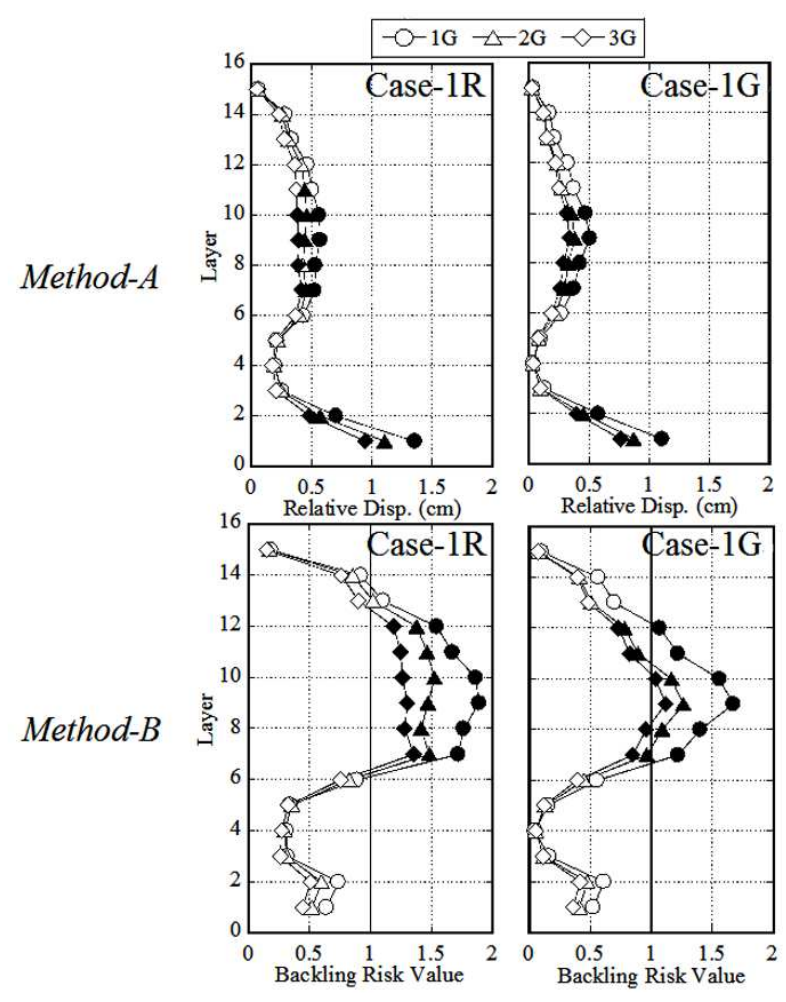

Fig. 15. Comparison with empirical methods

\section{Solution Space of SOP2 and SOP3}

A full search of the solution space of SOP2 and SOP3 is also performed. Fig. 13 shows the evaluated values of all solution candidates and the best solution of SOP2 and SOP3 represented as $\Delta$ and $\nabla$ respectively, which is called the global optimal solution. The solution distribution on the $x$-axis is divided into three groups (Groups A, B and C). Except for cases 1R and $3 R$, the global optimal solutions obtained by a full search are the same as the optimal solutions obtained with the SGA. For SOP3, global optimal solutions of cases $1 \mathrm{R}$ and $3 \mathrm{R}, \nabla$ in Fig. 13 (a1) and (a3) are separated from the evaluated values.

The VED arrangements of these solutions are series arrangements in 7th-12th layers. However, the errors between the evaluation values and analysis results reach $35 \%$ and the seismic performance is not necessarily excellent. This may be attributable to the fact that the vibration mode is altered after the VED replacement.The VED arrangements of the best and worst solutions of the maximum buckling risk index among Groups A and $\mathrm{C}$ of Fig. 13 (a1) are shown in Fig. 14. In solutions of Group A, the VEDs are arranged in the lower part of the truss tower, which contributes to reduce the maximum displacement of the top of the truss tower. In superior solutions of the buckling risk index (A-1 and C-1), members at 7th-12th layers, which are exposed to a higher risk of buckling than other layers, are replaced by VEDs.

\section{Comparison with Empirical Damper Arrangement Method}

Finally, the empirical damper design and the proposed optimal arrangement method are compared and the effectiveness of the empirical design method is investigated.

In Takeuchi (2005), two damper arrangement methods for a truss structure were proposed. The former method focuses on member deformation and the members connecting to nodes which produce relatively large are replaced with dampers (Method A). The other method focuses on the buckling risk index and the high-risk members in the pushover or elastic time history analysis are replaced with dampers (Method B). The effectiveness of these empirical design methods are investigated and compared with the proposed optimal solutions.

The maximum analysis result of the empirical design method obtained from the dynamic analysis of the nonretrofitted model in elastic state is shown in Fig. 15. The six largest design values are illustrated as the black marks and the VEDs are arranged in these layers. Based on Method B, members are replaced to the VEDs in 7th12th layers and these are the same results as those of the global solution in cases $1 \mathrm{R}$ and $3 \mathrm{R}$ of SOP3 (C-1, C-2 in Fig. 14). The seismic performances of these global solutions are not superior to that of the other cases. However, the locations of VED based on Method A are 1st, 2nd and 7th-11th layers similar to the optimal solutions of SOP2 and SOP3, which display the high correspondence between the evaluation values and the time history analysis results.

\section{Conclusion}

In this study, optimal arrangements of energy dissipating devices are investigated using the response spectrum with the equivalent linearization method and a genetic algorithm. The proposed method was applied to the viscoelastic damper arrangement in telecommunication tower structures. The obtained conclusions are summarized as follows:

- The response prediction rule based on a serial system provides accurate results of the maximum displacement of truss structure, taking high damping of the VEDs

- In the optimization problem of minimizing the maximum displacement of the truss tower at the top layer, the column and diagonal members of the lower part of the truss tower are mainly replaced to the VEDs

- In the optimization in the case of replacing only diagonal members, the location of the VEDs distributes mainly in two parts; the lower part of 
the truss tower contributes to the displacement response reduction and the middle part contributes to prevent members from buckling

- It is proven by the full search of the solution space of optimization problems SOP2 and SOP3 that the proposed optimization method with an SGA generally results in the global optimal solution except in some specific cases

- The solutions based on the empirical design method focusing on the relative displacement gives similar results to the optimal solution of SOP2 and SOP3, which proves the effectiveness of the displacement based empirical design method

Although second and third conclusions are considered to be specific characteristics of analysis models in this study, the other conclusions are considered to be valid with other truss structures. Optimal solutions have mostly similar results under various ground motions; therefore, the optimal design solutions obtained using the proposed method or the empirical design method based on the relative displacement of members may show superior potential under various ground motions. Accordingly, these design proposals are considered to be useful in general damper design applications. The applicability of this optimization method to different types of structures will be investigated and the generality of the optimal design methodology will be discussed in future studies.

\section{Funding Information}

The authors have no support or funding to report.

\section{Author's Contributions}

Toru Takeuchi and Yusuke Kinouchi: Organized research plan, carried out optimal analyses and contributed to writing of the manuscript.

Ryota Matsui and Toshiyuki Ogawa: Provided technical consultancy about numerical analyses and discussions.

\section{Ethics}

Author declares that there are not ethical issues that may arise after the publication of this manuscript.

\section{References}

Papageorgiou1, A.V. and C.J. Gantes, 2010a. Decoupling criteria for inelastic irregular primary/secondary structural systems subject to seismic excitation. J. Eng. Mechanics, 136: 1234-1247.

DOI: 10.1061/(ASCE)EM.1943-7889.0000166
Papageorgiou1, A.V. and C.J. Gantes, 2010b. Equivalent modal damping ratios for concrete/steel mixed structures. Comput. Structures, 88: 1124-1136. DOI: 10.1016/j.compstruc.2010.06.014

Hagishita, T. and M. Ohsaki, 2008. Optimal placement of braces for steel frames with semi-rigid joints by scatter search. Comput. Structures, 86: 1983-1993. DOI: 10.1016/j.compstruc.2008.05.002

Holland, J.H., 1975. Adaptation in Natural and Artificial Systems. Ann Arbor, Univ. of Michigan Press,

Kasai, K., Y. Fu and A. Watanabe, 1998. Passive control systems for seismic damage mitigation. J. Structural Eng., 124: 501-512. DOI: 10.1061/(ASCE)0733-9445(1998)124:5(501)

Nakazawa, S., H. Murakami, S. Kato, Y. Ookouchi and T. Takeuchi et al., 2006. Study on a retrofit method of telecommunication steel towers using buckling restrained braces: Application of a grid computing system for optimal device arrangements based on a genetic algorithm. J. Structural Construction Eng. Transaction AIJ, 604: 79-86.

Ogawa, T., T. Takeuchi, M. Nakagawa and T. Kumagai, 2003. Seismic response analysis of single layer lattice domes with substructure. IASS-APCS Int. Symposium Proc.

Ookouchi, Y., T. Takeuchi, T. Uchiyama, K. Suzuki and T. Sugiyama et al., 2006. Experimental studies of tower structures with hysteretic dampers. J. Int. Association Shell Spatial Structures, 47: 229-236.

Takeuchi, T., K. Kasai, K. Ohara, Y. Kimura and H. Nakashima, 2002. Performance evaluation and design of passively controlled building using equivalent linearization. Structural Eng. World Congress.

Takeuchi, T., 2005. Design of space truss structures with damping member (in Japanese). Japan Steel Structure J., 18: 22-29.

Wilson, E.L., A.D. Kiureghian and E.P. Bayo, 1981. A replacement for the SRSS method in seismic analysis. Earthquake Eng. Structural Dynamics, 9: 187-192. DOI: 10.1002/eqe.4290090207

Young, K. and H. Adeli, 2014. Fundamental period of irregular concentrically braced steel frame structures. Structural Design Tall Special Buildings, 23: 1211-1224. DOI: 10.1002/tal.1136 